\title{
Staphylococcus muscae, a New Species Isolated from Flies
}

\author{
V. HÁJEK, ${ }^{1 *}$ W. LUDWIG, ${ }^{2}$ K. H. SCHLEIFER, ${ }^{2}$ N. SPRINGER, ${ }^{2}$ W. ZITZELSBERGER, ${ }^{2}$ \\ R. M. KROPPENSTEDT, ${ }^{3}$ AND M. KOCUR ${ }^{4}$ \\ Department of Microbiology, Faculty of Medicine, Palacký University, 77515 Olomouc, Czechoslovakia ${ }^{1}$; \\ Lehrstuhl für Mikrobiologie, Technische Universität München, D-8000 Munich, Germany²; Deutsche Sammlung \\ von Mikroorganismen und Zellkulturen, D-3300 Braunschweig, Germany ${ }^{3}$; and Czechoslovak Collection of \\ Microorganisms, Masaryk University, Brno, Czechoslovakia ${ }^{4}$
}

\begin{abstract}
A new coagulase-negative species of the genus Staphylococcus, Staphylococcus muscae, is described on the basis of the results of a study of four strains that were isolated from flies. 16S rRNA sequences of the type strains of S. muscae, Staphylococcus schleiferi, and Staphylococcus sciuri were determined and used, together with the corresponding sequences of Staphylococcus aureus and Staphylococcus epidermidis, for a comparative analysis. The new species is characterized taxonomically; this species is differentiated from the other novobiocin-susceptible staphylococci by its physiological and biochemical activities, cell wall composition, and levels of genetic relatedness. The type strain of this species is strain MB4 (= CCM 4175).
\end{abstract}

A total of 28 species are currently recognized in the genus Staphylococcus; 19 species are listed in Bergey's Manual of Systematic Bacteriology (14), two species (Staphylococcus chromogenes and Staphylococcus lentus) have been elevated from the subspecies level $(8,19)$, and 7 species have been described since 1984 (Staphylococcus arlettae, Staphylococcus delphini, Staphylococcus equorum, Staphylococcus felis, Staphylococcus kloosii, Staphylococcus lugdunensis, and Staphylococcus schleiferi) $(5,11,21,26)$. Most of the new species were isolated from animals; the exceptions were $S$. lugdunensis and $S$. schleiferi, which were isolated from humans. The descriptions of all 28 Staphylococcus species were based on studies of strains that were isolated from mammals, birds, and foodstuffs of animal origin.

In this paper, we describe a new coagulase-negative species of staphylococci that was obtained from the body surfaces of flies. These organisms were found in about $7 \%$ of the flies caught in certain cow sheds but not on flies caught in human dwellings, stables, or piggeries (7). For this reason, this bacterium should be regarded as a transient rather than a resident on flies.

In this report we present phenetic and molecular taxonomic data for a new species, Staphylococcus muscae.

\section{MATERIALS AND METHODS}

Bacterial strains. Four strains were isolated from flies trapped in cow sheds; strains $\mathrm{MB} 4^{\mathrm{T}}$, $(\mathrm{T}=$ type strain $)$, MB21, and MB50 were isolated from Stomoxys calcitrans, and strain MB30 was isolated from Musca domestica (7). The medium used for isolation was blood agar base no. 2 (Oxoid) supplemented with $5 \%$ defibrinated ovine blood. Nutrient agar (Oxoid) containing glucose $(2 \%, \mathrm{wt} / \mathrm{vol})$ and $\mathrm{P}$ agar (15) were used to propagate all of the isolates. Inocula for tests were prepared from 1-day cultures that were incubated at $37^{\circ} \mathrm{C}$. All strains were stored as frozen suspensions in glycerol broth at $-25^{\circ} \mathrm{C}(10)$ and also in a freezedried state.

Methods. The procedures used for determining morphological, physiological, and biochemical characteristics have been described elsewhere (6). The type strains of the 14 novobiocin-susceptible Staphylococcus species (see Table 2)

\footnotetext{
* Corresponding author.
}

were obtained from the Deutsche Sammlung von Mikroorganismen (DSM), Braunschweig, Germany, the American Type Culture Collection (ATCC), Rockville, Md., and the Czechoslovak Collection of Microorganisms (CCM), Brno, Czechoslovakia.

Phage typing was carried out by using the method of Blair and Williams (1). We used 24 phages (25) for human Staphylococcus aureus strains, 12 phages (24) for bovine $S$. aureus strains, 4 experimental phages (2) for Staphylococcus intermedius strains, and 4 phages (9) for Staphylococcus hyicus strains.

We used the methods described by Schleifer and Kandler (20) and Schleifer (18) for cell wall preparation and determination. Cellular fatty acids were determined by gas chromatography, using the method of Miller (17). DNA base composition was determined by thermal denaturation with a model 2600 spectrophotometer (Gilford Instruments Laboratories, Inc., Oberlin, Ohio) (3). DNA from Escherichia coli K-12 was used as a standard (13). Radioactive labeling of DNA and the DNA-DNA hybridization experiments were carried out by using the filter method as described previously (12). 16S rRNA genes (16S rDNA) were amplified in vitro by using the polymerase chain reaction as described previously by Ludwig et al. (16). The amplified $16 \mathrm{~S}$ rDNA fragment was cloned in vector pBluescript (Stratagene, La Jolla, Calif.) and $E$. coli JM83. The cloned DNA was sequenced by using the chain termination method in combination with sitespecific primers (16). Sequences of 16S rRNA genes were aligned, similarities between sequence pairs were determined, and an unrooted phylogenetic tree was constructed by using the method of Fitch and Margoliash (4) as described by Schleifer et al. (23).

\section{RESULTS AND DISCUSSION}

The four strains which we investigated were gram-positive, catalase-positive cocci that occurred in irregular clumps, were facultatively anaerobic, were susceptible to furadantin, and were resistant to bacitracin. The DNA base compositions of the four strains ranged from 40 to $41 \mathrm{~mol} \%$ guanine plus cytosine (strains $\mathrm{MB}^{\mathrm{T}}$ and MB21, $40 \mathrm{~mol} \%$; strains MB30 and MB50, $41 \mathrm{~mol} \%$ ); these values are somewhat higher than the values obtained for the previously described staphylococci ( 30 to $39 \mathrm{~mol} \%$ ). The fatty acid compositions of three $S$. muscae strains are summarized in 


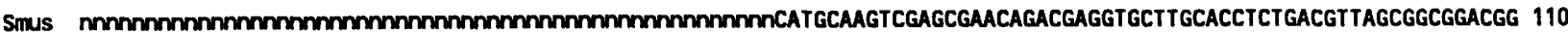
Ssch nnnnnnnnnnnmmnnnnnnGATGAACGCTGGCGGCGTGCCTAATACATGCAAGTCGAGCGAACGGACGAGGAGCTTGCTCCTTTGAAGTTAGCGGCGGACGG Ssci nnnmmmnnmmmmmmmmnGATGAACGCTGGCGGCGTGCCTAATACATGCAAGTCGAGCGACAGATGAGAGCTTGCTTCTCTGATGTTAGCGGCGGACGG

Smus GTGAGTAACACGTGGGTAACCTACCTATAAGACTGGAATAACTTCGGGAACCGGAGCTAATGCCGGATAATATATTGAACCGCATGGTTCAATAGTGAAAGACGGTTIT 220 Ssch GTGAGTAACACGTGGGTAACCTACCTATAAGACTGGATTAACTCCGGGAAACCGGGGCTAATGCCGGATAACATGTTGAACCGCATGGTTCAACAGTGAAAGACGGTCTT Ssci GTGAGTAACACGTGGGTAACCTACCTATAAGACTGGGATAACTCCGgGAACCGGGGCTAATACCGGATAATATITTGAACCGCATGGTTCAATAGTGAAAGACGGTITC

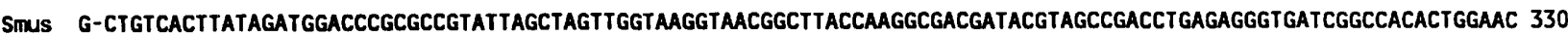
Ssch G-CTGTCACTTATAGATGGACCCGCGCCGTATTAGCTAGTTGGTGGGGTAACGGCCTACCAAGGCGACGATACGTAGCCGACCTGAGAGGGTGATCGGCCACACTGGAAC Ssci GGCTGTCACTTATAGATGGACCCGCGCCGTATTAGCTAGTTGgTAagGTAACGGCTTACCAAGGCGACGATACGTAGCCGACCTGAGAGGGTGATCGGCCACACTGGAAC

Smus TGAGACACGGTCCAGACTCCTACGGGAGGCAGCAGTAGGGAATCTTCCGCAATGGGCGAAAGCCTGACGGAGCAACGCCGCGTGAGTGATGAAGGTCTTCGGATCGTAAA 440 Ssch TGAGACACGGTCCAGACTCCTACGGGAGGCAGCAGTAGGGAATCTTCCGCAATGGGCGAAAGCCTGACGGAGCAACGCCGCGTGAGTGATGAAGGTCTICGGATCGTAAA Ssci TGAGACACGGTCCAGACTCCTACGgGAGGCAGCAGTAGGGAATCTTCCGCAATGGGCGAAGGCCTGACGGAGCAACGCCGCGTGAGTGATGAAGGTCTICGGATCGTAAA

Smus GCTCTGtTATtAGgGAaGAACAAACGTGTAAGTAACTGTGCACGTCTTGACGGTACCTAATCAGAAAGCCACGGCTAACTACGTGCCAGCAGCCGCGGTAATACGTAGGT 550 Ssch GCTCTGTTGTTAGgGAagAaCAAATGTGTAAGTAACTGTGCACGTCTTGACGGCACCTAACCAGAAGGCACGGCTAACTACGTGCCAGCAGCCGCGGTAATACGTAGGT Ssci ACTCTGTTGTTAGgGaAGAaCAAATTTGTTAGTAACTGAACAAGTCTTGACGGTACCTAACCAGAMGCCACGGCTAACTACGTGCCAGCAGCCGCGGTAATACGTAGGT

Smus GGCAAGCGTTATCCGGAATTATTGGGCGTAAAGCGCGCGTAGGCGGTTITTTAAGTCTGATGTGAAAGCCCACGGCTCAACCGTGGAGGGTCATTGGAAACTGGAAAACT 660 Ssch GGCAGCGTTATCCGGAATTATTGGGCGTAAAGCACGCGTAGGCGGTTITTTAAGTCTGATGTGAAAGCCCACGGCTCAACCGTGGAGGGTCATTGGAAACTGGAAAACT Ssci GGCAAGCGTTATCCGGAATTATTGGGCGTAAAGCGCGCGTAGGCGGTTTCTTAAGTCTGATGTGAAGGCCCACGGCTCAACCGTGGAGGGTCATTGGAAACTGGGAAACT

Smus TGAGTGCAGAAGAGgAaGTGGAATTCCATGTGTAGCGgTGAAATGCGCAGAGATATGGAGGAACACCAGTGGCGAaGGCGGCTITCTGGTCTGCAACTGACGCTGATGT 770 Ssch TGAGTGCAGAaGAGGAAAGTGGMTTCCATGTGTAGCGGTGAAATGCGCAGAGATATGGAGGAACACCAGTGGCGAAGGCGGCTTTCTGGTCTGCAACTGACGCTGATGT

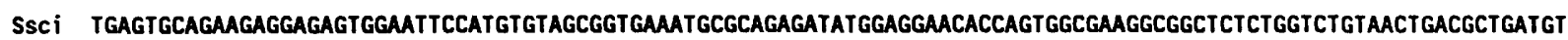

Smus GCGAAAGCGTGGGGATCAAACAGGATTAGATACCCTGGTAGTCCACGCCGTAAACGATGAGTGCTAAGTGTTAGGGGGTTTCCGCCCCTTAGTGCTGCAGCTAACGCATT 880 Ssch GCGAAAGCGTGGGGATCAAACAGGATTAGATACCCTGGTAGTCCACGCCGTAAACGATGAGTGCTAAGTGTTAGGGGGTTTCCGCCCCTIAGTGCTGCAGCTAACGCATT Ssci GCGAAAGCGTGGGGATCAAACAGGATTAGATACCCTGGTAGTCCACGCCGTAAACGATGAGTGCTAAGTGTTAGGGGGTTTCCGCCCCTTAGTGCTGCAGCTAACGCATI

Smus AAGCACTCCGCCTGGGGAGTACGGTCGCAAGACTGAAACTCAAAGGAATTGACGGGGACCCGCACAAGCGGTGGAGCATGTGGITTAATTCGAAGCAACGCGAAGAACCT 990 Ssch AAGCACTCCGCCTGGGGAGTACGGTCGCAAGACTGAAACTCAAAGGAATTGACGGGGACCCGCACAAGCGGTGGAGCATGTGGITIAATICGAAGCAACGCGAAGAACCT Ssci AAGCACTCCGCCTGGGGAGTACGACCGCAMGGTTGAAACTCAAAGGAATTGACGGGGACCCGCACAAGCGGTGGAGCATGTGGTTTAATTCGAAGCAACGCGAAGAACCT

Smus taCCAAATCTTGACATCCTITGACCGCACTAGAGATAGTGTTTTCCTCTTCGGAGGACAAAGTGACAGGTGGTGCATGGTTGTCGTCAGCTCGTGTCGTGAGATGTTGGG 1100 Ssch TACCAAATCTTGACATCCTTTGACCGCTCTAGAGATAGAGTTTTCCTCTTCGGAGGACAAAGTGACAGGTGGTGCATGGTTGTCGTCAGCTCGTGTCGTGAGATGTTGGG SsCi TACCAAATCTIGACATCCTITGACCGCTCTAGAGATAGAGTCTTCCCCTICGGGGGACAAAGTGACAGGTGGTGCATGGTTGTCGTCAGCTCGTGTCGTGAGATGITGGG

Smus TTAAGTCCCGCAACGAGCGCAACCCTIGAGCTIAGTTGCCATCATTAAGTTGGGCACTCTAAGTTGACTGCCGGTGACAAACCGGAGGAaGGTGGGGATGACGTCAAATC 1210 Ssch TTAAGTCCCGCAACGAGCGCAACCCTTGAGCTTAGTTGCCATCATTAAGTTGGGCACTCTAAGT TGACTGCCGGTGACAAACCGGAGGAAGGTGGGGATGACGTCAAATC Ssci TTAAGTCCCGCAACGAGCGCAACCCTTAAGCTTAGTTGCCATCATTAGGTTGGGCACTCTAGGTTGACTGCCGGTGACAAACCGGAGGAAGGTGGGGATGACGTCAAATC

Smus AICATGCCCCTTATGATITGGGCTACACACGTGCTACAATGGACATTACAAAGGGCAGCGAAACCGCGAGGTCAAGCAAATCCCATAAAGTTGITCTCAGTTCGGATIGT 1320 Ssch ATCATGCCCCTTATGATtTGGGCTACACACGTGCTACAATGGACAATACAMAGGGCAGCGAAACCGCGAGCTCAAGCAAATCCCATAAAGTTGTTCTCAGTTCGGATTGT Ssci ATCATGCCCCTTATGATtTGgGCTACACACGTGCTACAatggatAATACAAAGgGCAGCGAATCCGCGAGGCCAAGCAAATCCCATAAAATTATTCTCAGTTCGGATTGT

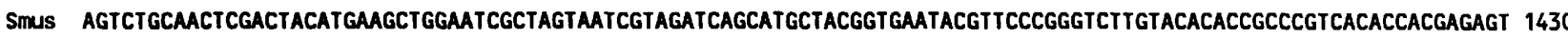
Ssch AGTCTGCMACTCGACTACATGAAGCTGGAATCGCTAGTAATCGTAGATCAGCATGCTACGGTGAATACGTTCCCGGGTCTTGTACACACCGCCCGTCACACCACGAGAGT Ssci AGTCTGCAACGCGACTACATGAAGCTGGAATCGCTAGTAATCGTAGATCAGCATGCTACGGTGAATACGTTCCCGgGTCTTGTACACACCGCCCGTCACACCACGAGAGT

Smus TTGTAACACCCGAAGCCGGTGGAGTAACCATTT-GGAGCTAGCCGTCGAAGGTGGGACAAATGATTGGGGTGAAGTCGTAACAAGGTAGCCGTATCGGAAGGTGCGGCTG 1540 Ssch TTGTAACACCCGAAGCCGGTGGAGTAACCATITIGGAGCTAGCCGTCGAAGGTGGGACAAATGATIGGGGTGAAGTCGTAACAAGGTAGCCGTATCGGAAGGTGCGGCTG Ssci TTGTAACACCCGAAGCCGGTGGAGTAACCTTITAGGAGCTAGCCGTCGAAGGTGGGACAAATGATTGGGGTGAAGTCGTAACAAGGTAGCCGTATCGGAAGGTGCGGCTG

Smus GATCACCTCCTTTCT 1555

SSCh GATCACCTCCTTTCT

Ssci GATCACCTCCTTTCT

FIG. 1. Nucleotide sequences of $16 \mathrm{~S}$ rRNAs of $S$. muscae (Smus), S. sciuri (Ssci), and S. schleiferi (Ssch). Bases which were not determined are indicated by $n$. 
TABLE 1. Fatty acid compositions of $S$. muscae strains

\begin{tabular}{|c|c|c|c|c|c|c|c|c|c|c|c|}
\hline \multirow{2}{*}{ Strain } & \multicolumn{11}{|c|}{ Fatty acids composition $(\%)^{a}$} \\
\hline & iso- $\mathrm{C}_{11}$ & iso- $C_{13}$ & $\mathrm{C}_{14: 0^{b}}$ & iso- $\mathrm{C}_{15}$ & anteiso- $\mathrm{C}_{15}$ & $\mathrm{C}_{16: 0}$ & iso- $\mathrm{C}_{17}$ & anteiso- $\mathrm{C}_{17}$ & $\mathrm{C}_{18: 0}$ & iso- $\mathrm{C}_{19}$ & $\mathrm{C}_{20: 0}$ \\
\hline $\mathrm{MB} 4^{\mathrm{T}}$ & $\operatorname{tr}^{c}$ & 1.1 & 3.7 & 40.9 & 1.0 & 14.8 & 5.8 & 1.9 & 10.7 & 1.0 & 10.1 \\
\hline MB21 & 0.4 & 1.8 & 7.8 & 40.6 & 9.7 & 18.3 & 4.3 & 1.5 & 9.4 & 0.6 & 5.5 \\
\hline MB30 & 0.3 & 1.9 & 7.9 & 34.6 & 9.7 & 23.6 & 2.7 & 1.1 & 10.9 & 0.4 & 6.3 \\
\hline
\end{tabular}

" Data are expressed as percentages of the total fatty acid methyl esters

$b$ 14:0, 14 carbons in straight-chain saturated (tetradecanoic) acid.

${ }^{c}$ Trace (less than $0.25 \%$ ).

Table 1. In particular, the presence of long-chain saturated unbranched fatty acid $\mathrm{C}_{20: 0}$ ( $n$-eicosanoic acid) is typical of staphylococci (22). DNA-DNA hybridization studies showed that the four strains are genetically closely related to one another but not to any of the other staphylococcal species (Table 2). 16S rRNA gene fragments from strain MB4 ${ }^{\mathrm{T}}$ and the type strains of S. schleiferi and Staphylococcus sciuri were amplified, cloned, and sequenced; the sequences are aligned in Fig. 1. A comparison of these sequences and those of S. aureus and Staphylococcus epidermidis (15a) indicated that strain $\mathrm{MB} 4^{\mathrm{T}}$ is certainly a member of the genus Staphylococcus and is slightly more closely related to $S$. schleiferi than to the other staphylococci whose $16 \mathrm{~S}$ rRNA sequences are known (Fig. 2). The cell walls contained a glycine-rich peptidoglycan, which is typical of staphylococci. Strains $\mathrm{MB}^{\mathrm{T}}$ and MB21 contained peptidoglycan type Lys-Gly ${ }_{5-6}$, and strain MB50 contained peptidoglycan type Lys-Gly ${ }_{4}$, Ser. Moreover, the phosphate contents of the cell walls (450 to $900 \mathrm{nmol}$ of phosphate per $\mathrm{mg}$ of cell wall) and the occurrence of glycerol in cell wall hydrolysates indicated that a cell wall teichoic acid was present in all four strains (3a).

Description of Staphylococcus muscae sp. nov. The description of the new species Staphylococcus muscae (mus'cae. L. gen. n. musca, of a fly) was based on the test results obtained with strains $\mathrm{MB}^{\mathrm{T}}$, MB21, MB30, and MB50.

All of the strains which we studied are small gram-positive cocci that range from 0.4 to $1.1 \mu \mathrm{m}$ in diameter and are arranged predominantly in irregular clumps, occasionally in pairs and singly. Spherical or slightly ovoid cells are nonmotile and nonsporeforming.

Colonies on $\mathrm{P}$ agar (after 5 days at $37^{\circ} \mathrm{C}$ ) are only slightly convex with gently raised centers, circular, entire, smooth,

TABLE 2. Levels of DNA-DNA relatedness between S. muscae strains ${ }^{a}$

\begin{tabular}{lcccc}
\hline $\begin{array}{c}\text { Source of } \\
\text { unlabeled DNA }\end{array}$ & \multicolumn{4}{c}{$\%$ of homology with labeled DNA from strain: } \\
\cline { 2 - 5 } & $\mathrm{MB}^{\mathrm{T}}$ & $\mathrm{MB} 21$ & $\mathrm{MB} 30$ & MB50 \\
\hline S. muscae MB4 & $100^{\mathrm{T}}$ & 100 & 85 & 90 \\
S. muscae MB21 & 100 & 100 & 90 & ND \\
S. muscae MB30 & 95 & 95 & 100 & ND \\
\hline
\end{tabular}

${ }^{a}$ The values were determined under optimal hybridization conditions $\left(25^{\circ} \mathrm{C}\right.$ below the melting temperature of the DNA). We also determined the levels of relatedness between each of the $S$. muscae strains and the following other Staphylococcus strains: $S$. arlettae DSM 20672 ${ }^{\mathrm{T}}, S$. aureus ATCC $12600^{\mathrm{T}}, S$. auricularis DSM $20609^{\mathrm{T}}$, S. caprae CCM $3573^{\mathrm{T}}$, S. carnosus DSM $20501^{\mathrm{T}}$, S. cohnii DSM $20260^{\mathrm{T}}, S$. equorum DSM $20674^{\mathrm{T}}, S$. gallinarum DSM $20610^{\mathrm{T}}, S$. hyicus ATCC $11249^{\mathrm{T}}, S$. intermedius CCM $5739^{\mathrm{T}}, S$, kloosii DSM $20676^{\mathrm{T}}, S$. lentus ATCC $29070^{\mathrm{T}}, S$. lugdunensis DSM $4804^{\mathrm{T}}$, and $S$. schleiferi DSM $4807^{\mathrm{T}}$. The values which we obtained were all less than $15 \%$.

$b$ Percent relatedness. ND, not determined. faintly glistening, butyrous, opaque, grayish white, and 5 to $6 \mathrm{~mm}$ in diameter.

These organisms grow well in the presence of $\mathrm{NaCl}$ at concentrations up to $10 \%$; no growth occurs at an $\mathrm{NaCl}$ concentration of $15 \%$. They grow moderately at $25^{\circ} \mathrm{C}$; no growth occurs at 10 and $45^{\circ} \mathrm{C}$.

Facultative anaerobes; better growth occurs under aerobic conditions. Anaerobic growth in semisolid thioglycolate medium is evident after 24 to $48 \mathrm{~h}$.

All of the strains produce catalase, phosphatase (strain MB50 weakly), and heat-labile nuclease and are positive in benzidine tests and weakly positive in methyl red tests. These organisms exhibit clear hemolysis on ovine blood agar medium (strains MB21 and MB30 weakly). All of the strains produce lecithinase, split Tween 20, Tween 40, and Tween 80 , and reduce nitrates. On crystal violet agar, three of them produce white (positive, E type) colonies, while strain MB30 produces blue (negative, D type) colonies.

None of the strains produces oxidase, coagulase, clumping factor, fibrinolysin, thermostable nuclease, tellurite reductase, gelatinase, protease, urease, arginine dihydrolase, alpha- or beta-hemolysins, ornithine decarboxylase, acetylmethylcarbinol, or beta-galactosidase. The strains do not hydrolyze starch or esculin.

The strains produce acid aerobically from glucose (without gas), fructose, sucrose, trehalose, turanose, xylose, and glycerol. No acid is produced from adonitol, arabinose, arbutin, cellobiose, dulcitol, fucose, galactose, inositol, inulin, lactose, maltose, mannitol, mannose, melezitose, melibiose, raffinose, rhamnose, ribose, salicin, sorbose, or tagatose. All of the strains except strain MB30 have the ability to produce acid anaerobically from glucose weakly.

All of the strains are resistant to lysozyme (MIC, $>1,000$ $\mu \mathrm{g} / \mathrm{ml}$ ) and susceptible to novobiocin (MIC, 0.06 to 0.1 $\mu \mathrm{g} / \mathrm{ml}$ ), penicillin (MIC, 0.01 to $0.06 \mu \mathrm{g} / \mathrm{ml}$ ), oxacillin (MIC, $0.2 \mu \mathrm{g} / \mathrm{ml}$ ), ampicillin (MIC, 0.06 to $1.0 \mu \mathrm{g} / \mathrm{ml}$ ), cephaloridine

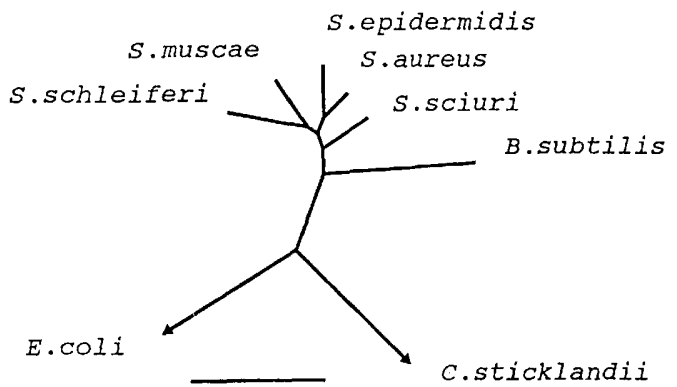

FIG. 2. Phylogenetic relationships of $S$. muscae. The tree was constructed by using distance values $\left(K_{\text {nuc }}\right)(23)$. Bar $=0.05 K_{\text {nuc }}$. B., Bacillus; C., Clostridium. 
TABLE 3. Characteristics that differentiate $S$. muscae from other novobiocin-susceptible Staphylococcus species

\begin{tabular}{|c|c|c|c|c|c|c|c|c|c|c|c|c|c|c|c|}
\hline Characteristic & $\begin{array}{c}\tilde{\Xi} \\
\text { క్ } \\
\Xi \\
\dot{s}\end{array}$ & 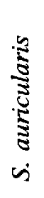 & $\begin{array}{l}\frac{3}{3} \\
\bar{\Xi} \\
\dot{s}\end{array}$ & 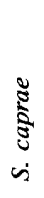 & 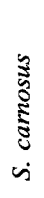 & 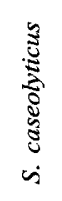 & 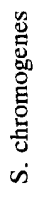 & 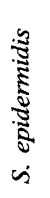 & $\underset{\dot{s}}{\stackrel{\tilde{z}}{\tilde{E}}}$ & 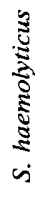 & 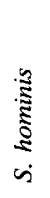 & 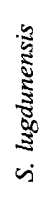 & 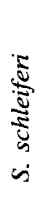 & 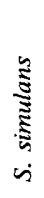 & 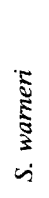 \\
\hline Pigment & $-^{a}$ & - & - & - & - & $\mathrm{v}$ & + & - & - & $\mathrm{v}$ & $\mathrm{v}$ & $\mathrm{v}$ & - & - & $\mathrm{v}$ \\
\hline Oxidase & - & - & - & - & - & + & - & - & - & - & - & - & - & - & - \\
\hline Alkaline phosphatase & + & - & - & + & + & - & + & + & + & - & - & - & + & $\mathrm{w}$ & - \\
\hline Urease & - & - & - & + & - & ND & $\mathrm{v}$ & + & + & - & + & $\mathbf{v}$ & - & + & + \\
\hline Hemolysis & $\mathbf{w}$ & - & $\mathbf{v}$ & + & - & - & - & $\mathrm{v}$ & $\mathbf{v}$ & + & $\mathrm{v}$ & w & $\mathbf{w}$ & $\mathbf{v}$ & $\mathrm{v}$ \\
\hline Ornithine & - & - & - & - & - & - & - & - & - & - & - & + & - & - & - \\
\hline Clumping factor & - & - & - & - & - & ND & - & - & - & - & - & + & + & - & - \\
\hline Heat-stable nuclease & - & - & - & - & $\mathbf{v}$ & ND & - & - & - & - & - & - & + & - & - \\
\hline Acetoin & - & $\mathrm{v}$ & $\mathrm{v}$ & + & + & - & - & + & - & + & $v$ & + & + & - & + \\
\hline \multicolumn{16}{|l|}{ Acid produced from: } \\
\hline Lactose & - & - & - & + & - & + & + & $\mathrm{v}$ & + & $\mathbf{v}$ & $\mathbf{v}$ & $\mathbf{v}$ & - & + & $v$ \\
\hline Maltose & - & $\mathrm{v}$ & - & $\mathrm{v}$ & - & + & $\mathrm{v}$ & + & - & + & + & + & - & - & $\mathbf{v}$ \\
\hline Mannitol & - & - & + & - & + & - & $\mathrm{v}$ & - & $\mathbf{v}$ & $\mathbf{v}$ & - & - & - & + & $\mathbf{v}$ \\
\hline Mannose & - & - & + & + & + & - & + & $\mathrm{v}$ & + & - & - & + & + & $\mathbf{v}$ & - \\
\hline Ribose & - & - & - & - & + & + & + & $\mathrm{v}$ & $\mathbf{v}$ & $\mathbf{v}$ & - & - & - & $\mathrm{v}$ & $\mathbf{v}$ \\
\hline Saccharose & + & $\mathrm{v}$ & $\mathrm{v}$ & - & - & $\mathrm{v}$ & + & + & $\mathbf{v}$ & + & + & + & - & + & + \\
\hline Trehalose & + & + & - & + & $\mathrm{v}$ & + & + & - & + & + & + & + & $\mathrm{v}$ & $\mathrm{v}$ & + \\
\hline Turanose & + & $\mathrm{v}$ & - & - & - & - & $\mathrm{v}$ & $\mathrm{v}$ & ND & $\mathrm{v}$ & $\mathbf{v}$ & $\mathbf{w}$ & - & - & - \\
\hline Xylose & + & - & - & - & - & - & - & - & - & - & - & - & - & - & - \\
\hline
\end{tabular}

${ }^{a}+$, positive; $\mathbf{w}$, weak; $\mathbf{v}$, variable; -, negative; ND, not determined.

(MIC, 0.1 to $0.2 \mu \mathrm{g} / \mathrm{ml}$ ), erythromycin (MIC, $0.06 \mu \mathrm{g} / \mathrm{ml}$ ), lincomycin (MIC, 0.2 to $0.5 \mu \mathrm{g} / \mathrm{ml}$ ), clindamycin (MIC, 0.1 $\mu \mathrm{g} / \mathrm{ml}$ ), chloramphenicol (MIC, $2.0 \mu \mathrm{g} / \mathrm{ml}$ ), tetracycline (MIC, 0.1 to $0.2 \mu \mathrm{g} / \mathrm{ml}$ ), gentamicin (MIC, $0.2 \mu \mathrm{g} / \mathrm{ml}$ ), and vancomycin (MIC, $1.0 \mu \mathrm{g} / \mathrm{ml})$. All four strains are resistant to bacitracin (10 U per disc) and susceptible to furadantin (100 $\mu \mathrm{g}$ per disc) when the disc diffusion method is used.

The strains are resistant to all of the phages which we used at $100 \times$ routine test dilution. The dominant cellular fatty acids are iso- $\mathrm{C}_{15: 0}$ (13 methyltetradecanoic) and $\mathrm{C}_{16: 0}$ (hexadecanoic) acids (Table 1 ).

The peptidoglycan type of strains $\mathrm{MB}^{\mathrm{T}}$ and MB21 is Lys-Gly $_{5-6}$, and the peptidoglycan type of strain MB50 is Lys-Gly, Ser. None of the strains have protein A.

The average guanine-plus-cytosine content of the DNA is 40 to $41 \mathrm{~mol} \%$.

Strain MB4 (= CCM 4175) is the type strain of S. muscae. The characteristics of this strain are the same as those given above for the species and in Table 1.

The phenotypic properties that are useful for differentiating $S$. muscae from the other coagulase-negative staphylococcal species are shown in Table 3. S. muscae can be distinguished from the other species on the basis of its susceptibility to novobiocin, positive test for alkaline phosphatase, negative tests for oxidase, acetoin, pigment, and urease, carbohydrate reaction pattern, peptidoglycan type, and comparatively high DNA base composition.

\section{REFERENCES}

1. Blair, J. E., and R. E. O. Williams. 1961. Phage typing of staphylococci. Bull. W. H. O. 24:771-784.

2. Blouse, L., and W. E. Meekins. 1968. Isolation and use of experimental phages for typing Staphylococcus aureus isolates from sentry dogs. Am. J. Vet. Res. 29:1817-1822.

3. DeLey, J. 1970. Re-examination of the association between melting point, buoyant density, and chemical base composition of deoxyribonucleic acid. J. Bacteriol. 101:738-754.

3a.Fiedler, F. Personal communication.
4. Fitch, W. M., and E. Margoliash. 1967. Construction of phylogenetic trees. Science 155:279-284.

5. Freney, J., Y. Brun, M. Bes, H. Meugnier, F. Grimont, P. A. D. Grimont, C. Nervi, and J. Fleurette. 1988. Staphylococcus lugdunensis sp. nov. and Staphylococcus schleiferi sp. nov., two species from human clinical specimens. Int. J. Syst. Bacteriol. 38:168-172.

6. Hájek, V. 1976. Staphylococcus intermedius, a new species isolated from animals. Int. J. Syst. Bacteriol. 26:401-408.

7. Hájek, V., and J. Balasek. 1985. Staphylococci from flies of different environments. Zentralbl. Bakteriol. Parasitenkd. Infektionskr. Hyg. Abt. 1 Suppl. 14:129-133.

8. Hájek, V., L. A. Devriese, M. Mordarski, M. Goodfellow, G. Pulverer, and P. E. Varaldo. 1986. Elevation of Staphylococcus hyicus subsp. chromogenes (Devriese et al., 1978) to species status: Staphylococcus chromogenes (Devriese et al., 1978) comb. nov. Syst. Appl. Microbiol. 8:169-173.

9. Hájek, V., and V. Horák. 1981. Typing of staphylococci with phages derived from Staphylococcus hyicus. Zentralbl. Bakteriol. Parasitenkd. Infektionskr. Hyg. Abt. 1 Suppl. 10:93-98.

10. Howard, D. H. 1956. The preservation of bacteria by freezing in glycerol broth. J. Bacteriol. 71:625.

11. Igimi, S., S. Kawamura, E. Takahashi, and T. Mitsuoka. 1989. Staphylococcus felis, a new species from clinical specimens from cats. Int. J. Syst. Bacteriol. 39:373-377.

12. Kilpper-Bälz, R., U. Buhl, and K. H. Schleifer. 1980. Nucleic acid homology studies between Peptococcus saccharolyticus and various anaerobic and facultatively anaerobic gram-positive cocci. FEMS Microbiol. Lett. 8:205-210.

13. Kilpper-Bälz, R., P. Wenzig, and K. H. Schleifer. 1985. Molecular relationships and classification of some viridans streptococci as Staphylococcus oralis and emended description of Staphylococcus oralis (Bridge and Sneath, 1982). Int. J. Syst. Bacteriol. 35:482-488.

14. Kloos, W. E., and K. H. Schleifer. 1986. Genus IV. Staphylococcus Rosenbach 1884, 18 $8^{\mathrm{AL}}$, (Nom. Cons. Opin. $17 \mathrm{Jud}$. Comm. 195, 153), p. 1013-1035. In P. H. A. Sneath, N. S. Mair, M. E. Sharpe, and J. G. Holt (ed.), Bergey's manual of systematic bacteriology, vol. 2. The Williams \& Wilkins Co., Baltimore.

15. Kloos, W. E., T. G. Tornabene, and K. H. Schleifer. 1974. 
Isolation and characterization of micrococci from human skin, including two new species: Micrococcus lylae and Micrococcus kristinae. Int. J. Syst. Bacteriol. 24:79-101.

15a.Lane, D., and E. Stackebrandt. Personal communication

16. Ludwig, W., M. Weizenegger, S. Dorn, J. Andreesen, and K. H. Schleifer. 1990. The phylogenetic position of Peptococcus niger based on 16S rRNA sequence studies. FEMS Microbiol. Lett. 71:139-144.

17. Miller, L. T. 1982. A single derivatization method for bacterial fatty acid methyl esters, including hydroxy acids. J. Clin. Microbiol. 16:584-586.

18. Schleifer, K. H. 1985. Analysis of the chemical composition and primary structure of murein, p. 123-156. In G. Gottschalk (ed.), Methods in microbiology, vol. 18. Academic Press, Inc. (London), Ltd., London.

19. Schleifer, K. H., U. Geyer, R. Kilpper-Bälz, and L. A. Devriese. 1983. Elevation of Staphylococcus sciuri subsp. lentus (Kloos et al.) to species status: Staphylococcus lentus (Kloos et al.) comb. nov. Syst. Appl. Microbiol. 4:382-387.

20. Schleifer, K. H., and O. Kandler. 1972. Peptidoglycan types of bacterial cell walls and their taxonomic implications. Bacteriol. Rev. 36:407-477.

21. Schleifer, K. H., R. Kilpper-Bälz, and L. A. Devriese. 1984. Staphylococcus arlettae sp. nov., S. equorum sp. nov., and $S$. kloosii sp. nov.: three new coagulase-negative, novobiocin- resistant species from animals. Syst. Appl. Microbiol. 5:501509.

22. Schleifer, K. H., and R. M. Kroppenstedt. 1990. Chemical and molecular classification of staphylococci. J. Appl. Bacteriol. 69:9-24.

23. Schleifer, K. H., M. Leuteritz, N. Weiss, W. Ludwig, G. Kirchhof, and H. Seidel-Rüffer. 1990. Taxonomic study of anaerobic, gram-negative, rod-shaped bacteria from breweries: emended description of Pectinatus cerevisiphilus and description of Pectinatus frisingensis sp. nov., Selenomonas lacticifex sp. nov., Zymophilus raffinosivorans gen. nov., sp. nov., and Zymophilus paucivorans sp. nov. Int. J. Syst. Bacteriol. 40:1927.

24. Subcommittee on Phage-Typing of Staphylococci. 1971. Report of the Subcommittee on Phage-Typing of Staphylococci to the International Committee on Nomenclature of Bacteria (19661970). Int. J. Syst. Bacteriol. 21:167-170.

25. Subcommittee on Phage-Typing of Staphylococci. 1975. Report of the Subcommittee on Phage-Typing of Staphylococci to the International Committee on Systematic Bacteriology (19701974). Int. J. Syst. Bacteriol. 25:241-242.

26. Varaldo, P. E., R. Killper-Bälz, F. Biavasco, G. Satta, and K. H. Schleifer. 1988. Staphylococcus delphini sp. nov., a coagulasepositive species isolated from dolphins. Int. J. Syst. Bacteriol. 38:436-439. 